\title{
Quantum effects on curve crossing in a Bose-Einstein condensate
}

\author{
V. A. Yurovsky ${ }^{1,2,3}$, A. Ben-Reuven ${ }^{2}$, and P. S. Julienne ${ }^{3}$ \\ ${ }^{1}$ ITAMP, Harvard-Smithsonian Center for Astrophysics, 60 Garden Street, Cambridge MA 02138 \\ ${ }^{2}$ School of Chemistry, Tel Aviv University, 69978 Tel Aviv, Israel \\ ${ }^{3}$ Atomic Physics Division, Stop 8423, National Institute of Standards and Technology, Gaithersburg, MD 20889
}

(November 6, 2018)

\begin{abstract}
Formation of atomic pairs by the dissociation of a molecular condensate or by inelastic collisions in an atomic condensate due to a time-dependent curve crossing process is studied beyond the mean-field approximation. The number of atoms formed by the spontaneous process is described by a Landau-Zener formula multiplied by an exponential amplification factor due to quantum many-body effects. The atomic pairs are formed in an entangled (squeezed) state. The rate of stimulated processes depends on the relative phase of the
\end{abstract} two fields.

03.75.Fi, 03.65.Nk, 34.50.Lf, 34.50.Rk

Introduction. - The concept of curve crossing was introduced in the pioneering works of Landau [1] and Zener [2] concerning transitions between two linear potentials. Curve-crossing theory has many applications in various fields of physics (see, e.g., Ref. [3] and references therein), usually describing transitions between states whose energies cross as a function of the time (a non-stationary crossing) or some coordinate (a stationary crossing).

Recent developments in the physics of Bose-Einstein condensates (BEC) (see Refs. [.5] and references therein) demand taking account of many-body effects in a curve crossing. These effects, which cannot be described by the usual mean-field method, are treated here by a second-quantized formalism. Hereafter we call a process involving such effects a "many-body crossing", as distinct from a "single-body crossing" in which a colliding pair of atoms is treated as a single body (a quasimolecule).

A major many-body effect on curve-crossing in BEC is Bose enhancement. In the photoassociation of atomic BEC this effect leads to Bose-stimulated Raman adiabatic passage (see Ref. [6] and references therein). An expression for the transition probability as a function of the rate of energy variation was obtained in $[7,8]$ by treating the process as a curve crossing event in a nonlinear system. The nonlinearity may lead to a deviation of the transition probability from that predicted by the wellknown Landau-Zener (LZ) formula.

Although the association of atoms into molecules has been described within a mean-field approach (using coupled Gross-Pitaevskii equations), the process of dissociation of molecules into the atomic vacuum

$$
A_{2} \rightarrow A+A
$$

cannot be described by this approach, since the coupling is proportional to the atomic field, the initial value of which is zero. Therefore, at least initially, the dissociation must be a spontaneous process, which is neglected in the mean-field approach.

The time-independent dissociation of a molecular BEC was considered in Ref. [9] by using a numerical solution of the full quantum equations. It is shown there that the initial stage of the dissociation can be approximately described with good accuracy by analytical expressions obtained by treating the molecular field as a mean field, while the atomic field is treated as a second-quantized field. This method is similar to the parametric approximation used in quantum optics (see Ref. [10]). This approximation is valid as long as the population of the molecular state is large and the effect of its depletion can be neglected.

In the present paper we generalize the parametric approximation to the case of time-dependent condensate energies. The energies can be varied by applying an external time-dependent magnetic field or by changing the frequency of laser fields coupling the states. In general, the dissociation of a molecular condensate requires taking into account the coupling to multiple atomic modes, in a manner analogous to methods of multistate curve crossing theory [3, 11]. Here we concentrate on the essential physics by considering only a single atomic mode, showing how quantum effects lead to a substantial initial enhancement of the dissociation and to the formation of atoms in squeezed states that are entangled (see also 12 (14).

Similar quantum effects can also play an important role in the association of atomic condensates, where the molecular state is coupled to excited atomic trap states (which are initially not populated). Transitions to these states can substantially affect the condensate loss rate [8,15] and therefore should also be treated by a multimode model using a quantum approach similar to the one described below.

The effects of Bose enhancement and the formation of entangled atoms should also appear in an atomic BEC, if inelastic collisions of the type

$$
A(0)+A(0) \rightarrow A+A,
$$

where $A(0)$ and $A$ denote different internal states of the atoms, change the states of both colliding atoms. Such a process might be realized by applying an electromagnetic field, or two fields (as in a Raman process), in res- 
onance with the two-atom transition, provided that the concurrent one-atom transition $(A(0) \rightarrow A)$ is off resonance with the applied fields. The time variation of the energy can again be due to the Zeeman effect or to time-dependent electromagnetic field frequencies. An experimental observation of Bose enhancement and entanglement may be simpler in an atomic BEC than in the molecular one.

General crossing. - Consider a system of coupled atomic $A$ and molecular $A_{2}$ fields, associated with the annihilation operators $\hat{\psi}$ and $\hat{\psi}_{m}$, respectively. In the case of one atomic and one molecular mode the system can be described by the Hamiltonian

$$
\hat{H}=\epsilon(t) \hat{\psi}^{\dagger} \hat{\psi}+g_{\mathrm{am}} \hat{\psi}_{m}^{\dagger} \hat{\psi} \hat{\psi}+g_{\mathrm{am}}^{*} \hat{\psi}^{\dagger} \hat{\psi}^{\dagger} \hat{\psi}_{m}
$$

where $\epsilon(t)$ is a time-dependent energy of the atomic state, while the energy of the molecular state is chosen as the zero energy. The parameter $g_{\text {am }}$ describes the coupling of the atomic and molecular fields due to hyperfine interaction or laser fields.

The parametric approximation used in Ref. [9] for the case of constant $\epsilon$ can be generalized to the case of a time-dependent $\epsilon$, by retaining the assumption of a large molecular population. We can then replace the operator $\hat{\psi}_{m}$ by its expectation value $\left\langle\hat{\psi}_{m}\right\rangle$, and obtain the equation of motion for the atomic field operator $\hat{\psi}$ :

$$
i \dot{\hat{\psi}}=\epsilon(t) \hat{\psi}+2 g^{*} \hat{\psi}^{\dagger}
$$

(using units in which $\hbar=1$ ), where

$$
g=g_{\mathrm{am}}\left\langle\hat{\psi}_{m}\right\rangle^{*}
$$

Let us also consider two atomic states, $A(0)$ and $A$, associated with the field operators $\hat{\psi}_{0}$ and $\hat{\psi}$, respectively. Taking into account one mode for each of the states, one can describe the system by the following Hamiltonian

$$
\hat{H}=\epsilon(t) \hat{\psi}^{\dagger} \hat{\psi}+g_{\text {aa }} \hat{\psi}_{0}^{\dagger} \hat{\psi}_{0}^{\dagger} \hat{\psi} \hat{\psi}+g_{\text {aa }}^{*} \hat{\psi}^{\dagger} \hat{\psi}^{\dagger} \hat{\psi}_{0} \hat{\psi}_{0}
$$

where $\epsilon(t)$ is the time-dependent energy of state $A$, while the energy of state $A(0)$ is chosen as the zero energy. Whenever both wavevectors $k_{0}$ and $k$, of the entrance and exit reaction channels (2), are in the near-threshold region, the inelastic scattering amplitude should have the form $T=\sqrt{k_{0} k} b$, where $b$ is an energy-independent length (see Ref. 16]). The parameter $g_{\text {aa }}$ can then be expressed as $g_{\text {aa }}=4 \pi b / \mathrm{m}$. The parametric approximation can be applied here if the state $A(0)$ has a large population, replacing the product $\hat{\psi}_{0} \hat{\psi}_{0}$ by its expectation value $\left\langle\hat{\psi}_{0} \hat{\psi}_{0}\right\rangle$. If the atoms are initially in a condensate (coherent) state, then $\left\langle\hat{\psi}_{0} \hat{\psi}_{0}\right\rangle=\left\langle\hat{\psi}_{0}\right\rangle^{2}$. Denoting

$$
g=g_{\mathrm{aa}}\left\langle\hat{\psi}_{0} \hat{\psi}_{0}\right\rangle^{*}
$$

one obtains again the same equation of motion (4) for the field operator $\hat{\psi}$.
The set of two first-order equations [Eq. (4) and its hermitian conjugate] can be transformed to a single secondorder equation

$$
\ddot{\hat{\psi}}+\left(\epsilon^{2}(t)-4|g|^{2}+i \dot{\epsilon}(t)\right) \hat{\psi}=0 .
$$

Although it is an operator equation, it is linear and its solution can be expressed as

$$
\hat{\psi}(t)=\psi_{c}(t) \hat{\psi}\left(t_{0}\right)+\psi_{s}(t) \hat{\psi}^{\dagger}\left(t_{0}\right),
$$

where $\psi_{c, s}(t)$ are two independent $c$-number solutions of Eq. (8). Equations (4) and (9) at $t=t_{0}$ give the initial conditions for $\psi_{c, s}\left(t_{0}\right)$ and their derivatives:

$$
\begin{aligned}
& \psi_{c}\left(t_{0}\right)=1, \quad \psi_{s}\left(t_{0}\right)=0, \\
& \dot{\psi}_{c}\left(t_{0}\right)=-i \epsilon\left(t_{0}\right), \quad \dot{\psi}_{s}\left(t_{0}\right)=-2 i g^{*} .
\end{aligned}
$$

The solutions $\psi_{c}(t)$ and $\psi_{s}(t)$ can be expressed in terms of a pair of conventionally selected independent solutions of Eq. (\$) $\psi_{1}(t)$ and $\psi_{2}(t)$, treated as "standard solutions", in the form

$$
\begin{aligned}
\psi_{c}(t)= & \frac{i}{W\left\{\psi_{1}, \psi_{2}\right\}}\left[\left(\epsilon\left(t_{0}\right) \psi_{2}\left(t_{0}\right)-i \dot{\psi}_{2}\left(t_{0}\right)\right) \psi_{1}(t)\right. \\
& \left.-\left(\epsilon\left(t_{0}\right) \psi_{1}\left(t_{0}\right)-i \dot{\psi}_{1}\left(t_{0}\right)\right) \psi_{2}(t)\right], \\
\psi_{s}(t)= & \frac{2 i g^{*}}{W\left\{\psi_{1}, \psi_{2}\right\}}\left[\psi_{2}\left(t_{0}\right) \psi_{1}(t)-\psi_{1}\left(t_{0}\right) \psi_{2}(t)\right],
\end{aligned}
$$

where $W\left\{\psi_{1}, \psi_{2}\right\}=\psi_{1} \dot{\psi}_{2}-\psi_{2} \dot{\psi}_{1}$ is the Wronskian of the standard solutions. In the special case of a timeindependent $\epsilon$ [9], $\psi_{s}(t)$ and $\psi_{c}(t)$ can be expressed in terms of sine and cosine functions (circular or hyperbolic, depending on the ratio of $g$ to $\epsilon$ ).

The use of Eq. (9) allows us to express the expectation value of any function of $\hat{\psi}^{\dagger}$ and $\hat{\psi}$ in terms of the expectation value at a particular time $t=t_{0}$. Thus, the number of atoms is a sum of two terms $\left\langle\hat{\psi}^{\dagger}(t) \hat{\psi}(t)\right\rangle=$ $n_{s p}(t)+n_{s t}(t)$, where the first term

$$
n_{s p}(t)=\left|\psi_{s}(t)\right|^{2}
$$

corresponds to spontaneous transitions into the vacuum of $\hat{\psi}$, and the second term

$$
\begin{aligned}
n_{s t}(t)= & \left(\left|\psi_{c}(t)\right|^{2}+\left|\psi_{s}(t)\right|^{2}\right)\left\langle\hat{\psi}^{\dagger}\left(t_{0}\right) \hat{\psi}\left(t_{0}\right)\right\rangle \\
& +2 \operatorname{Re}\left(\psi_{s}^{*}(t) \psi_{c}(t)\left\langle\hat{\psi}\left(t_{0}\right) \hat{\psi}\left(t_{0}\right)\right\rangle\right) .
\end{aligned}
$$

depicts stimulated transitions taking place when the final atomic state is initially populated. The first and second terms on the right-hand side of Eq. (15) correspond to a noncoherent and a coherent initial occupation, respectively. Consider also the expectation value of a generalized coordinate operator

$$
\hat{Q}(t)=\hat{\psi}(t) e^{i \theta}+\hat{\psi}^{\dagger}(t) e^{-i \theta}
$$


frequently used in the theory of squeezed states (see, e. g., [17]). We present here only an expression for the spontaneous process, in which $\left\langle\hat{\psi}^{\dagger}\left(t_{0}\right) \hat{\psi}\left(t_{0}\right)\right\rangle=$ $\left\langle\hat{\psi}\left(t_{0}\right) \hat{\psi}\left(t_{0}\right)\right\rangle=0$ :

$$
\left\langle\hat{Q}^{2}(t)\right\rangle_{s p}=\left|\psi_{c}(t) e^{i \theta}+\psi_{s}^{*}(t) e^{-i \theta}\right|^{2} .
$$

Linear crossing. - Single-body curve-crossing problems are commonly solved by using the LZ formula, which is an exact solution of the linear non-stationary problem. Let us consider a generalization to our case of a many-body crossing, choosing $\epsilon(t)$ as the linear function $\epsilon(t)=\beta t$. The second-order equation (8) attains in this case the form of a parabolic cylinder equation (see Ref. [18])

$$
\ddot{\hat{\psi}}+\left(\beta^{2} t^{2}-4|g|^{2}+i \beta\right) \hat{\psi}=0 .
$$

This equation differs from the corresponding c-number equation for a single-body crossing (see Ref. [11]) by the sign before $4|g|^{2}$ due to the effects of secondary quantization. An appropriate choice of a pair of standard solutions is then

$$
\begin{aligned}
& \psi_{1}(t)=U\left(\frac{1}{2}+i \lambda,-e^{-i \pi / 4} \tau\right), \\
& \psi_{2}(t)=\frac{1}{g} \sqrt{\beta / 2} e^{-i \pi / 4} U\left(-\frac{1}{2}-i \lambda,-e^{i \pi / 4} \tau\right),
\end{aligned}
$$

where $U(a, x)$ is the parabolic cylinder function, defined by Eq. (19.3.1) of Ref. [18. Here $\tau=\sqrt{2 \beta} t$, and

$$
\lambda=2|g|^{2} / \beta .
$$

The parameter $\lambda$ is nothing else but the LZ exponent, since the coupling of states, according to Eq. (4), is $2 g$ and the slope of the two-atom energy is $2 \beta$.

The solutions (19) have the Wronskian $W\left\{\psi_{1}, \psi_{2}\right\}=$ $i(\beta / g) \exp (-\pi \lambda / 2)$ and in addition obey the conditions

$$
i \dot{\psi}_{i}-\beta t \psi_{i}=2 g^{*} \psi_{3-i}^{*} \quad(i=1,2),
$$

which allow us to express $\psi_{c}(t)$ as

$$
\psi_{c}(t)=-2 i g^{*} \frac{\psi_{1}^{*}\left(t_{0}\right) \psi_{1}(t)-\psi_{2}^{*}\left(t_{0}\right) \psi_{2}(t)}{W\left\{\psi_{1}, \psi_{2}\right\}} .
$$

Using a common procedure of curve crossing theories, we can start by considering transitions during a finite time interval $\left[t_{0}, t\right]$, with $t_{0}<0<t$, assuming sufficiently large values of $\left|t_{0}\right|$ and $t$ (obeying the validity criteria presented below). Asymptotic expansions of the parabolic cylinder functions [18] give us at $t_{0} \rightarrow-\infty$

$$
\begin{aligned}
& \psi_{1}\left(t_{0}\right) \sim \frac{1}{\left|\tau_{0}\right|} \exp \left(-\frac{\pi}{4} \lambda+i \frac{\pi}{4}+i S\left(\left|\tau_{0}\right|\right)\right), \\
& \psi_{2}\left(t_{0}\right) \sim \frac{1}{g} \sqrt{\frac{\beta}{2}} \exp \left(-\frac{\pi}{4} \lambda-i \frac{\pi}{4}-i S\left(\left|\tau_{0}\right|\right)\right),
\end{aligned}
$$

where $\tau_{0}=\sqrt{2 \beta} t_{0}$ and $S(\tau)=\tau^{2} / 4-\lambda \ln \tau$. The asymptotic expansions at $t \rightarrow \infty$, obtained by using the relation (19.4.6) in [18], are given by

$$
\begin{aligned}
& \psi_{1}(t) \sim \frac{1}{\tau} \exp \left(\frac{3 \pi}{4} \lambda-i \frac{5 \pi}{4}+i S(\tau)\right) \\
& +\frac{1}{|g|} \sqrt{\beta \sinh (\pi \lambda)} \exp \left(\frac{\pi}{4} \lambda-i \frac{\pi}{2}-i S(\tau)-i \arg \Gamma(i \lambda)\right), \\
& \psi_{2}(t) \sim \frac{1}{g} \sqrt{\beta / 2} \exp \left(\frac{3 \pi}{4} \lambda-i \frac{\pi}{4}-i S(\tau)\right) \\
& +\frac{g^{*}}{|g| \tau} \sqrt{2 \sinh (\pi \lambda)} \exp \left(\frac{\pi}{4} \lambda-i \frac{\pi}{2}+i S(\tau)+i \arg \Gamma(i \lambda)\right) .
\end{aligned}
$$

Spontaneous process. - Substituting the asymptotic expansions, Eqs. (23) and (24), at large $\left|t_{0}\right|$ and $t$ into Eqs. (13) and (14), one can obtain the number of atoms formed by the spontaneous process

$$
\begin{aligned}
n_{s p}(t) \sim\left(e^{2 \pi \lambda}-1\right)+ & 2 e^{\pi \lambda} \sqrt{\lambda\left(e^{2 \pi \lambda}-1\right)} \\
\times & {\left[\frac{\cos \chi(\tau)}{\tau}-\frac{\cos \chi\left(\left|\tau_{0}\right|\right)}{\tau_{0}}\right], }
\end{aligned}
$$

where $\chi(\tau)=\tau^{2} / 2-2 \lambda \ln \tau+\arg \Gamma(i \lambda)$.

Equation (25) contains two terms of an asymptotic expansion in inverse powers of $\tau$ and $\tau_{0}$. The asymptotic approach is applicable if the second term in Eq. (25) is small compared to the first one, or

$$
t,\left|t_{0}\right| \gg|g / \beta|,
$$

in agreement with estimates of the transition region for two-state single-body crossings (see Ref. 11]).

Let us recall that all the analysis above is based on the assumption that the depletion of the initial (atomic or molecular) condensate state is negligibly small, applicable when $n_{s p}(t) \ll N$, or

$$
\lambda<\frac{1}{2 \pi} \ln N
$$

where $N$ is the initial number of atoms. Since $N \gg 1$ (in current experiments an atomic BEC contains $10^{4}$ to $10^{6}$ atoms) the present approach is applicable even for large values of $\lambda$ corresponding to adiabatic transitions.

The leading term of Eq. (25) can be written as

$$
n_{s p} \approx\left(1-e^{-2 \pi \lambda}\right) e^{2 \pi \lambda} .
$$

The first factor here is the familiar LZ probability, while the second one describes an amplification of the spontaneous process. This amplification is reminiscent of lasing, as the exponent can be interpreted as a product of a characteristic crossing time $(g / \beta)$ and an "amplification coefficient" $g$.

The LZ exponent $\lambda$ is itself enhanced, being proportional to $N$ [see Eqs. (5) and (20)]. This is also true for 
the association process, but unlike that process, the Bose enhancement introduced here by the amplification factor is unique to the dissociation process. As an example, consider the Na Feshbach resonances studied in Ref. [8]. The parameter $\lambda$ there can be of the order of unity, depending on the ramp speed, in which case one obtains an amplification factor of more than a 100.

A distinction between many-body and single-body crossings appears also in the interpretation of the crossing as scattering on a parabolic potential barrier. Indeed, on replacing the time by a coordinate, Eq. (8) is transformed into a corresponding one-dimensional stationary Schrödinger equation. In the single-body case the energy lies above the barrier (see Ref. [1]). However, in the many-body case, as one can see from Eq. (8), the energy lies below the the barrier, and the process involves barrier penetration. This leads to the replacement of the negative exponents by positive ones, and yields the first term of Eq. (25) in place of the LZ formula.

Using Eqs. (13), (22), (23), and (24), we can write Eq. (17) as

$$
\begin{aligned}
\left\langle\hat{Q}^{2}\right\rangle_{s p} & \sim \mid \sqrt{e^{2 \pi \lambda}-1} \\
& +\left.\exp (\pi \lambda-i \chi(\tau)-i \arg g+2 i \theta-i \pi / 2)\right|^{2}
\end{aligned}
$$

Substituting one of the two orthogonal phase angles $\theta=$ $\theta_{ \pm}=\frac{1}{2}(\chi(\tau)+\arg g) \pm \frac{\pi}{4}$ in Eq. (16) we obtain

$$
\left\langle\hat{Q}_{ \pm}^{2}\right\rangle_{s p} \sim\left|\sqrt{e^{2 \pi \lambda}-1} \pm e^{\pi \lambda}\right|^{2} \underset{\lambda \gg 1}{\approx}\left\{\begin{array}{l}
4 e^{2 \pi \lambda} \\
\frac{1}{4} e^{-2 \pi \lambda} .
\end{array}\right.
$$

Therefore, the spontaneous dissociation forms atoms in a squeezed state (similar to a squeezed state of light [17]) in which $\hat{Q}_{+}$has an increased uncertainty and $\hat{Q}_{-}$has a reduced uncertainty. A similar effect concerning the time-independent case has been described in Ref. [9].

Stimulated process. - Consider now the transition stimulated by a finite initial occupation of the final atomic state. Starting from a coherent state $|\alpha\rangle$ of the operator $\hat{\psi}$, such that $\hat{\psi}\left(t_{0}\right)|\alpha\rangle=\alpha|\alpha\rangle$, and using Eqs. (15), (23), and (24), one obtains

$$
\begin{aligned}
n_{s t} & \sim|\alpha|^{2} \mid \sqrt{e^{2 \pi \lambda}-1} \\
& +\left.\exp \left(\pi \lambda+i \chi\left(\tau_{0}\right)+i \arg g+2 i \arg \alpha+i \pi / 2\right)\right|^{2}
\end{aligned}
$$

It follows from this expression that the final atomic population is dependent on the phase of $\alpha$, lying in a range bounded by the maximal and minimal values

$$
n_{ \pm}=|\alpha|^{2}\left|\sqrt{e^{2 \pi \lambda}-1} \pm e^{\pi \lambda}\right|^{2} \underset{\lambda \gg 1}{\approx}|\alpha|^{2}\left\{\begin{array}{l}
4 e^{2 \pi \lambda} \\
\frac{1}{4} e^{-2 \pi \lambda}
\end{array}\right.
$$

at $\arg \alpha=-\frac{1}{2}\left(\chi\left(\left|\tau_{0}\right|\right)+\arg g\right) \mp \frac{\pi}{4}$, respectively. Since $\arg g$ contains the phase of the molecular field $\left\langle\hat{\psi}_{m}\right\rangle$ [see
Eq. (5)] or the atomic field $\left\langle\hat{\psi}_{0}\right\rangle$ [see Eq. (7)], the stimulated dissociation is indeed dependent on the phase difference between the two fields ( $\alpha$ and $\left\langle\hat{\psi}_{m}\right\rangle$ or $\left\langle\hat{\psi}_{0}\right\rangle$ ). This effect is reminiscent of the amplification or absorption of coherent light in laser-active media, and its dependence on the phase of the light.

Conclusions. - The parametric approximation allows us to obtain an analytical expression (28) for the number of atoms spontaneously formed by the crossing of linearly time-dependent energies of the atomic and the molecular states or two atomic states. Many-body effects lead to an enhancement factor in the LZ exponent, as well as to the multiplication of the LZ probability by the amplification factor in Eq. (28) describing a positive-exponential enhancement of the spontaneous process. The atoms are formed in a squeezed state. The number of atoms formed by the stimulated process depends on the relative initial phase of the two fields. Similar quantum effects should also apply in other cases in which particles are created in pairs. An experimental realization of the processes we discuss may allow production of an entangled atomic gas.

The authors are grateful to P. D. Drummond and K. V. Kheruntsyan for helpful discussions. This work was partially supported by the NSF through a grant for the ITAMP at Harvard University and the SAO.

[1] L. D. Landau, Phys. Z. Sowjetunion 2, 46 (1932).

[2] C. Zener, Proc. R. Soc. London Ser. A 137, 696 (1932).

[3] V. A. Yurovsky and A. Ben-Reuven, Phys. Rev A 63, 043404 (2001).

[4] A. S. Parkins and D. F. Walls, Phys. Rep. 303, 1 (1998).

[5] F. Dalfovo, S. Giorgini, L. P. Pitaevskii, and S. Stringari, Rev. Mod. Phys. 71, 463 (1999).

[6] M. Mackie, R. Kowalski, and J. Javanainen, Phys. Rev. Lett. 84, 3803 (2000).

[7] V. A. Yurovsky, A. Ben-Reuven, P. S. Julienne and C. J. Williams, Phys. Rev. A 60, R765 (1999).

[8] V. A. Yurovsky, A. Ben-Reuven, P. S. Julienne and C. J. Williams, Phys. Rev. A 62, 043605 (2000).

[9] A. Vardi, V. A. Yurovsky, and J. R. Anglin, Phys. Rev. A (submitted).

[10] M. O. Scully and M. S. Zubairy, Quantum Optics (University Press, Cambridge, 1997).

[11] M. S. Child, Molecular Collision Theory (Academic Press, London and New York, 1974).

[12] A. Vardi and J. R. Anglin, Phys. Rev. Lett. 86, 568 (2001).

[13] J. R. Anglin and A. Vardi, Phys. Rev. A, 64, 013605 (2001).

[14] U. V. Poulsen and K. Molmer, Phys. Rev. A 63, 023604 (2001).

[15] F. H. Mies, E. Tiesinga, and P. S. Julienne, Phys.Rev. A 
61, 022721 (2000).

[16] P. S. Julienne and F. H. Mies, J. Opt. Soc. Am. B 6, 2257 (1989); H. R. Sadeghpour et al., J. Phys. B 33, R93 (2000).

[17] L. Mandel and E. Wolf, Optical Coherence and Quantum Optics (University Press, Cambridge, 1995).

[18] Handbook of Mathematical Functions, edited by M. Abramowitz and I. E. Stegun (NBS, Washington, 1964). 